\title{
Background Modeling via Incremental Maximum Margin Criterion
}

\author{
C. Marghes, T. Bouwmans \\ Laboratoire MIA, University of La Rochelle, 17000 La Rochelle, France
}

\begin{abstract}
Subspace learning methods are widely used in background modeling to tackle illumination changes. Their main advantage is that it doesn't need to label data during the training and running phase. Recently, White et al. [1] have shown that a supervised approach can improved significantly the robustness in background modeling. Following this idea, we propose to model the background via a supervised subspace learning called Incremental Maximum Margin Criterion (IMMC). The proposed scheme enables to initialize robustly the background and to update incrementally the eigenvectors and eigenvalues. Experimental results made on the Wallflower datasets show the pertinence of the proposed approach.
\end{abstract}

\section{Introduction}

Many background subtraction methods have been developed in video-surveillance to detect moving objects [2][3][4]. These methods have different common steps: background modeling, background initialization, background maintenance and foreground detection. The background modeling describes the kind of model used to represents the background. Once the model has been chosen, the background model is initialized during a learning step by using $\mathrm{N}$ frames. Then, a first foreground detection is made and consists in the classification of the pixel as a background or as a foreground pixel. Thus, the foreground mask is applied on the current frame to obtain the moving objects. After this, the background is adapted over time following the changes which have occurred in the scene and so on. The last decade witnessed very significant contributions in background modeling via unsupervised subspace learning [5] due to their robustness to illumination changes. The first approach developed by Oliver et al. [6] consists in applying Principal Component Analysis (PCA) on N images to construct a background model, which is represented by the mean image and the projection matrix comprising the first $\mathrm{p}$ significant eigenvectors of PCA. In this way, foreground segmentation is accomplished by computing the difference between the input image and its reconstruction. The main limitation of this method appears for the background maintenance because it is computationally intensive to perform model updating using the batch mode PCA. Moreover without a mechanism of robust analysis, the outliers or foreground objects may be absorbed into the background model. In this context, some authors proposed different algorithms 
of incremental PCA. The incremental PCA proposed by Rymel et al. [7] need less computation but the background image is contamined by the foreground object. To solve this, Li et al. [8] proposed an incremental PCA which is robust in presence of outliers. However, when keeping the background model updated incrementally, it assigned the same weights to the different frames. Thus, clean frames and frames which contain foreground objects have the same contribution. The consequence is a relative pollution of the background model. To solve this, Skocaj et al. [9] used a weighted incremental and robust. The weights are different following the frame and this method achieved a better background model. However, the weights were applied to the whole frame without considering the contribution of different image parts to building the background model. To achieve a pixel-wise precision for the weights, Zhang and Zhuang [10] proposed an adaptive weighted selection for an incremental PCA. This method performs a better model by assigning a weight to each pixel at each new frame during the update. Wang et al. [11] used a similar approach using the sequential Karhunen-Loeve algorithm. Recently, Zhang et al. [12] improved this approach with an adaptive scheme. All these incremental methods avoid the eigen-decomposition of the high dimensional covariance matrix using approximation of it and so a low decomposition is allowed at the maintenance step with less computational load. However, these incremental methods maintain the whole eigenstructure including both the eigenvalues and the exact matrix. To solve it, Li et al. [13] proposed a fast recursive and robust eigenbackground maintenance avoiding eigen-decomposition. This method achieves similar results than the incremental PCA [8] at better frames rates. In another way, Yamazaki et al. [14] and Tsai et al. [15] proposed to use the Independent Component Analysis (ICA) which is a variant of PCA in which the components are assumed to be mutually statistically independent instead of merely uncorrelated. This stronger condition allows remove the rotational invariance of PCA, i.e. ICA provides a meaningful unique bilinear decomposition of two-way data that can be considered as a linear mixture of a number of independent source signals. The ICA model was tested on traffic scenes [14] and show robustness in changing background like illumination changes. Recently, Chu et al. [16] used a Non-negative Matrix Factorization algorithm to model dynamic backgrounds and Bucak et al. [17] preferred an Incremental version of the Non-negative Matrix Factorization (INMF) which presents similar performance than the incremental PCA [8]. In order to take into account the spatial information, Li et al. [18] used an Incremntal Rank-(R1,R2,R3) Tensor (IRT). Results [18] show better robustness to noise. The Table 1 shows an overview of the background modeling based on subspace learning.

However, these different approaches are unsupervised subspace learning methods. Indeed, it doesnt need to label data. Recently, White et al. [1] proved that the Gaussian Mixture Model (GMM) [19] gives better results when some coefficients are determined in a supervised way. Following this idea, we propose to use a supervised subspace learning for background modeling. Thus, the Maximum Margin Criterion (MMC) offers a nice framework. It was proposed by $\mathrm{Li}$ et al. [20] and it can outperform PCA and Linear Discriminant Analysis (LDA) on 
many classification tasks [21]. MMC search for the projection axes on which the data points of different classes are far from each other meanwhile where data points of the same class are close to each other. As the original PCA and LDA, MMC is a batch algorithm and so it requires that the data must be known in advance and be given once altogether. Recently, Yan et al. [22] have proposed incremental version of MMC which is suitable to update online the background model.

The rest of this paper is organized as follows: In the Section 2, we firstly remind the Incremental Maximum Margin Criterion (IMMC). In the Section 3, we present our method using subspace learning via IMMC for background modeling. Then, a comparative evaluation is provided in the Section 4. Finally, the conclusion is given in Section 5.

\begin{tabular}{|l|l|}
\hline \multicolumn{1}{|c|}{ Subspace Learning - Methods } & \multicolumn{1}{c|}{ Authors - Dates } \\
\hline Principal Components Analysis & \multicolumn{1}{|c|}{ Oliver et al. (1999) [6] } \\
Batch PCA & Rymel et al. (2004)[7] \\
Incremental PCA & Li et al. (2003)[8] \\
Incremental and Robust PCA & Skocaj et al. (2003)[9] \\
Weighted Incremental and Robust PCA & Zhang and Zhuang (2007)[10] \\
Adaptive Weighted Incremental and Robust PCA & \\
\hline Independent Component Analysis & Yamazaki et al. (2006)[14] \\
Batch ICA & Tsai and Lai (2009) [15] \\
Incremental ICA & \\
\hline Independent Component Analysis & Chu et al. (2010)[16] \\
Batch NMF & Bucak et al. (2007)[17] \\
Incremental NMF & Li et al. (2008)[18] \\
\hline Independent Component Analysis & \\
Incremental Rank-(R1,R2,R3) Tensor &
\end{tabular}

Table 1. Subpace Learning for background modeling: An Overview

\section{Incremental Maximum Margin Criterion (IMMC)}

This section reminds briefly the principle of IMMC developed in [22]. Suppose the data sample points $u(1), u(2), \ldots, u(N)$ are $d$-dimensional vectors, and $U$ is the sample matrix with $u(i)$ as its $i^{\text {th }}$ column. MMC [20] projects the data onto a lower-dimensional vector space such that the ratio of the inter-class distance to the intra-class distance is maximized. The goal is to achieve maximum discrimination and the new low-dimensional vector can be computed as $y=W^{T} u$ where $W \in \mathbf{R}^{d \times p}$ is the projection matrix from the original space of dimension $d$ to the low dimensional space of dimension $p$. So, MMC [20] aims to maximize the criterion:

$$
J(W)=W^{T}\left(S_{b}-S_{w}\right) W
$$


where

$$
\begin{aligned}
S_{b} & =\sum_{i=1}^{c} p_{i}\left(m_{i}-m\right)\left(m_{i}-m\right)^{T} \\
S_{w} & =\sum_{i=1}^{c} p_{i} E\left(u_{i}-m_{i}\right)\left(u_{i}-m_{i}\right)^{T}
\end{aligned}
$$

are called respectively the inter-class scatter matrix and the intra-class scatter matrix and $c$ is the number of classes, $m$ is the mean of all samples, $m_{i}$ is the mean of the samples belonging to class $i$ and $p_{i}$ is the prior probability for a sample belonging to class $i$. The projection matrix $W$ can be obtained by solving:

$$
\left(S_{b}-S_{w}\right) w=\lambda w
$$

To incrementally maximize the MMC criterion, Yan et al.[22] constraint $\mathrm{W}$ to unit vectors, i.e. $W=\left[w_{1}, w_{2}, \ldots w_{p}\right]$ and $w_{k}^{T} w_{k}=1$. Thus the optimization problem of $J(W)$ is transformed to:

$$
\max \sum_{k=1}^{p} w_{k}^{T}\left(S_{b}-S_{w}\right) w_{k}
$$

subject to $w_{k}^{t} w_{k}=1$ with $k=1,2, \ldots, p . W$ is the first $k$ leading eigenvectors of the matrix $S_{b}-S_{w}$ and the column vectors of $W$ are orthogonal to each other. Thus, the problem is learning the $p$ leading eigenvector of $S_{b}-S_{w}$ incrementally.

\subsection{Updating incrementally leading eigenvectors}

Let $C=S_{b}+S_{w}$ be the covariance matrix, then we have $J(W)=W^{T}\left(2 S_{b}-C\right) W$, $W \in \mathbf{R}^{d \times p}$. Then maximizing $J(W)$ means to find the $p$ leading eigenvectors of $2 S_{b}-C$.

The inter-class scatter matrix of step $n$ after learning from the first $n$ samples can be written as below,

$$
S_{b}(n)=\sum_{j=1}^{c} p_{j}(n)\left(m_{j}-m(n)\right)\left(m_{j}(n)-m(n)\right)^{T}
$$

and

$$
S_{b}=\lim _{n \rightarrow \infty} \frac{1}{n} \sum_{i=1}^{n} S_{b}(i)
$$

On the other hand,

$$
\begin{gathered}
C=E(u(n)-m)(u(n)-m)^{T} \\
=\lim _{n \rightarrow \infty} \frac{1}{n} \sum_{i=1}^{n}(u(n)-m(n))(u(n)-m(n))^{T}
\end{gathered}
$$


$2 S_{b}-C$ should have the same eigenvectors as $2 S_{b}-C+\theta I$ where $\theta$ is a positive real number and $I \in R^{d \times d}$. From (7) and (9) we have the following equation:

$$
2 S_{b}-C+\theta I=\lim _{n \rightarrow \infty} \frac{1}{n} \sum_{i=1}^{n} A(i)=A
$$

where $A(i)=2 S_{b}(i)-(u(i)-m(i))(u(i)-m(i))^{T}+\theta I, A=2 S_{b}-C+\theta I$.

The general eigenvector form is $A x=\lambda x$, where $x$ is the eigenvector of matrix $A$ corresponding to the eigenvalue $\lambda$. By replacing matrix $A$ with the MMC matrix at step $n$, an approximate iterative eigenvector computation formulation is obtained with $\nu=\lambda x$.

$$
\begin{aligned}
& \nu(n)=\frac{1}{n} \sum_{i=1}^{n}\left(2 \sum_{j=1}^{c} p_{j}(i) \Phi_{j}(i) \Phi_{j}(i)^{T}\right. \\
& \left.-(u(i)-m(i))(u(i)-m(i))^{T}+\theta I\right) x(i)
\end{aligned}
$$

where $\Phi_{j}(i)=m_{j}(i)-m(i), v(n)$ is the $\mathrm{n}$ step estimation of $v$ and $x(n)$ is the $\mathrm{n}$ step estimation of $x$. Once the estimation of $\nu$ is obtained, eigenvector $x$ can be directly computed as $x=\nu /\|\nu\|$. Let $x(i)=\nu(i-1) /\|\nu(i-1)\|$, then the incremental formulation is the following:

$$
\begin{aligned}
& \nu(n)=\frac{n-1}{n} \nu(n-1) \\
& \quad+\frac{1}{n}\left(2 \sum_{j=1}^{c} p_{j}(n) \alpha_{j}(n) \Phi_{j}(n)\right. \\
& -\beta(u(n)-m(n))+\theta \nu(n-1)) /\|\nu(n-1)\|
\end{aligned}
$$

where $\alpha_{j}(n)=\phi_{j}(n)^{T} \nu(n-1)$ and $\beta(n)=(u(n)-m(n))^{T} \nu(n-1), j=1,2, \ldots, c$. For initialization, $\nu(0)$ is equal to the first data sample.

\subsection{Updating incrementally the other eigenvectors}

To compute the $(j+1)^{t h}$ eigenvector, its projection is substracted on the estimated $j^{\text {th }}$ eigenvector from the data,

$$
u_{1_{n}}^{j+1}(n)=u_{1_{n}}^{j}(n)-\left(u_{1_{n}}^{j}(n)^{T} \nu^{j}(n)\right) \nu^{j}(n)
$$

where $u_{1_{n}}^{1}(n)=u_{1_{n}}(n)$. The same method is used to update $m_{i}^{j}(n)$ and $m^{j}(n)$, $i=1,2, \ldots, c$. Since $m_{i}^{j}(n)$ and $m^{j}(n)$ are linear combinations of $x_{l_{i}}^{j}(i)$, where $i=1,2, \ldots, k$, and $l_{i} \in \mathbf{1}, \mathbf{2}, \ldots$, C. $\Phi_{i}$ are linear combination of $m_{i}$ and $m$, for convenience, only $\Phi$ is updated at each iteration step by:

$$
\Phi_{l_{n}}^{j+1}(n)=\Phi_{l_{n}}^{j}(n)-\left(\Phi_{l_{n}}^{j}(n)^{T} \nu^{j}(n)\right) \nu^{j}(n)
$$

In this way, the time-consuming orthonormalization is avoided and the orthogonal is always enforced when the convergence is reached. 


\section{Application to background modeling}

The Figure 1 shows an overview of the proposed approach. The background modeling framework based on IMMC includes the following stages: (1) Background initialization via $\mathrm{MMC}$ using $\mathrm{N}$ frames $(N=30$ pratically) (2) Foreground detection (3) Background maintenance using IMMC. The steps (2) and (3) are executed repeatedly as time progresses.

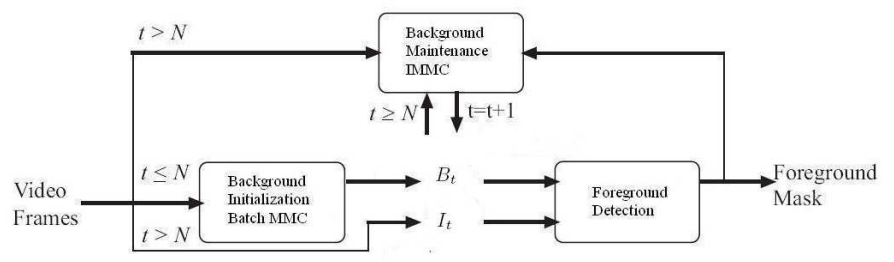

Fig. 1. Overview of the proposed approach

Denote the training video sequences $S=\left\{I_{1}, \ldots I_{N}\right\}$ where $I_{t}$ is the frame at time t. Let each pixel $(\mathrm{x}, \mathrm{y})$ be characterized by its intensity in the grey scale and asssume that we have the ground truth corresponding to this training video sequences, i.e we know for each pixel its class label which can be foreground or background. Thus, we have:

$$
\begin{aligned}
S_{b} & =\sum_{i=1}^{c} p_{i}\left(m_{i}-m\right)\left(m_{i}-m\right)^{T} \\
S_{w} & =\sum_{i=1}^{c} p_{i} E\left(u_{i}-m_{i}\right)\left(u_{i}-m_{i}\right)^{T}
\end{aligned}
$$

where $c=2, m$ is the mean of the intensity of the pixel $x, y$ over the training video and $m_{i}$ is the mean of samples belonging to class $i$ and $p_{i}$ is the prior probability for a sample belonging to class $i$ with $i \in\{$ Background, Foreground $\}$. Then, we can apply the batch MMC to obtain the first leading eigenvectors which correspond to the background. The corresponding eigenvalues are contained in the matrix $L_{M}$ and the leading eigenvectors in the matrix $\Phi_{M}$. Once the leading eigenbackground images stored in the matrix $\Phi_{M}$ are obtained and the mean $\mu_{B}$ too, the input image $I_{t}$ can be approximated by the mean background and weighted sum of the leading eigenbackgrounds $\Phi_{M}$.

So, the coordinate in leading eigenbackground space of input image $I_{t}$ can be computed as follows:

$$
w_{t}=\left(I_{t}-\mu_{B}\right)^{T} \Phi_{M}
$$

When $w_{t}$ is back projected onto the image space, a reconstructed background image is created as follows:

$$
B_{t}=\Phi_{M} w_{t}^{T}+\mu_{B}
$$


Then, the foreground object detection is made as follows:

$$
\left|I_{t}-B_{t}\right|>T
$$

where $\mathrm{T}$ is a constant threshold.

Once the first foreground detection is made, we apply the IMMC to update the background model using (12) and (14). The class label for each pixel is obtained using the foreground mask.

Remark: Note that the IMMC can be applied directly at time $t=1$ but it is less robust than to use firstly the batch algorithm on $\mathrm{N}$ frames and then to apply the IMMC to update the background.

\section{Experimental Results}

For the performance evaluation, we have compared our supervised approach with the unsupervised subspace learning methods PCA, INMF and IRT using the Wallflower dataset provided by Toyama et al. [23]. This dataset consists in a set of images sequences where each sequence presents a different type of difficulty that a practical task may meet: Moved Object (MO), Time of Day (TD), Light Switch (LS), Waving Trees (WT), Camouflage (C), Bootstrapping (B) and Foreground Aperture (F). The performance is evaluated against handsegmented ground truth. Three terms are used in evaluation: False Positive (FP) is the number of background pixels that are wrongly marked as foreground; False Negative (FN) is the number of foreground pixels that are wrongly marked as background; Total Error (TE) is the sum of FP and FN. The Table 2 shows the performance in term of FP, FN and TE for each algorithm. The corresponding results are shown in Table 3. As we can see, the IMMC gives the lowest TE followed by the IRT, the INMF and the PCA. Secondly, we have compared our supervised approach with the state of the art algorithms: SG[24], MOG[25] and KDE[26]. As we can see on the Table 2 and Table 3, our algorithm gives better results particularly in the case of illumination changes. The results for SG, MOG and PCA comes from [27]. The results for the INMF was provided by their authors [17]. The KDE was implemented in Microsoft Visual C++ and the IRT and IMMC was implemented in Matlab.

\section{Conclusion}

In this paper, we have proposed to model the background using a supervised subspace learning called Incremental Maximum Criterion. This approach allows to initialize robustly the background and to upate incrementally the eigenvectors and eigenvalues. Experimental results made on the Wallflower datasets show the pertinence of the proposed approach. Indeed, IMMC outperforms the supervised PCA, INMF and IRT. For future investigations, supervised subspace learning methods such as Linear Discriminant Analysis (LDA) and Canonical Correlation Analysis (CCA) seem to be very interesting approaches. For example, LDA exists 
in several incremental versions as incremental LDA using fixed point method [28] or sufficient spanning set approximations [29]. In the same way, Partial Least Squares (PLS) methods [30] give a nice perspective to model robustly the background.

\begin{tabular}{|c|c|c|c|c|c|c|c|c|c|}
\hline & & \multicolumn{7}{|c|}{ Problem Type } \\
\hline Algorithm & $\begin{array}{c}\text { Error } \\
\text { Type }\end{array}$ & MO & TD & LS & WT & C & B & FA & $\begin{array}{c}\text { Total } \\
\text { Errors (TE) }\end{array}$ \\
\hline SG & False neg & 0 & 949 & 1857 & 3110 & 4101 & 2215 & 3464 & \\
Wren et al.[24] & False pos & 0 & 535 & 15123 & 357 & 2040 & 92 & 1290 & 35133 \\
\hline MOG & False neg & 0 & 1008 & 1633 & 1323 & 398 & 1874 & 2442 & \\
Stauffer et al.[25] & False pos & 0 & 20 & 14169 & 341 & 3098 & 217 & 530 & 27053 \\
\hline KDE & False neg & 0 & 1298 & 760 & 170 & 238 & 1755 & 2413 & \\
Elgammal et al.[26] & False pos & 0 & 125 & 14153 & 589 & 3392 & 933 & 624 & 26450 \\
\hline PCA & False neg & 0 & 879 & 962 & 1027 & 350 & 304 & 2441 & \\
Oliver et al.[6] & False pos & 1065 & 16 & 362 & 2057 & 1548 & 6129 & 537 & 17677 \\
\hline INMF & False neg & 0 & 724 & 1593 & 3317 & 6626 & 1401 & 3412 & \\
Bucak et al.[17] & False pos & 0 & 481 & 303 & 652 & 234 & 190 & 165 & 19098 \\
\hline IRT & False neg & 0 & 1282 & 2822 & 4525 & 1491 & 1734 & 2438 & \\
\hline Li et al.[18] & False pos & 0 & 159 & 389 & 7 & 114 & 2080 & 12 & 17053 \\
\hline IMMC & False neg & 0 & 1336 & 2707 & 4307 & 1169 & 2677 & 2640 & \\
Proposed method & False pos & 0 & 11 & 16 & 6 & 136 & 506 & 203 & 15714 \\
\hline
\end{tabular}

Table 2. Performance Evaluation on Wallflower dataset[23]

\section{References}

1. White, B., Shah, M.: Automatically tuning background subtraction parameters using particle swarm optimization. ICME 2007 (2007) 1826-1829

2. Elhabian, S., El-Sayed, K., Ahmed, S.: Moving object detection in spatial domain using background removal techniques - state-of-art. RPCS 1 (Jan. 2008) 32-54

3. Bouwmans, T., Baf, F.E., Vachon, B.: Statistical background modeling for foreground detection: A survey. Handbook of Pattern Recognition and Computer Vision, World Scientific Publishing 4 (Jan. 2010) 181-189

4. Bouwmans, T., Baf, F.E., Vachon, B.: Background modeling using mixture of gaussians for foreground detection: A survey. RPCS 1 (Nov. 2008) 219-237

5. Bouwmans, T.: Subspace learning for background modeling: A survey. RPCS 2 (Nov. 2009) 223-234

6. Oliver, N., Rosario, B., Pentland, A.: A bayesian computer vision system for modeling human interactions. ICVS 1999 (Jan. 1999)

7. Rymel, J., Renno, J., Greenhill, D., Orwell, J., Jones, G.: Adaptive eigenbackgrounds for object detection. ICIP 2004 (Oct. 2004) 1847-1850

8. Li, Y., Xu, L., Morphett, J., Jacobs, R.: An integrated algorithm of incremental and robust pca. ICIP 2003 (Sept. 2003) 245-248 


\begin{tabular}{|c|c|c|c|c|c|c|c|}
\hline Sequence & $\mathrm{MO}$ & TD & $\mathrm{LS}$ & WT & $\mathrm{C}$ & $\mathrm{B}$ & FA \\
\hline Frame & $\begin{array}{c}\text { Frame } \\
985\end{array}$ & $\begin{array}{c}\text { Frame } \\
1850\end{array}$ & $\begin{array}{c}\text { Frame } \\
1865\end{array}$ & $\begin{array}{c}\text { Frame } \\
247\end{array}$ & $\begin{array}{c}\text { Frame } \\
251\end{array}$ & $\begin{array}{c}\text { Frame } \\
299\end{array}$ & 449 \\
\hline Test Imag & $-2 \pi$ & $=$ & & & & & \\
\hline Ground Tru & & & & & & & \\
\hline SG [24] & & & & & & & \\
\hline MOG [25] & & & & & & & \\
\hline KDE [26] & & & & & & & \\
\hline PCA [6] & & & & & & & \\
\hline INMF $[17$ & & Dis & & & & & \\
\hline IRT [18] & & $\rightarrow$ & & & & & \\
\hline IMMC & & & & & & & \\
\hline
\end{tabular}

Table 3. Results on Wallflower dataset[23] 
9. Skocaj, D., Leonardis, A.: Weighted and robust incremental method for subspace learning. ICCV 2003 (2003) 1494-1501

10. Zhang, J., Zhuang, Y.: Adaptive weight selection for incremental eigen-background modeling. ICME 2007 (Jul. 2007) 851-854

11. Wang, L., Wang, L., Zhuo, Q., Xiao, H., Wang, W.: Adaptive eigenbackground for dynamic background modeling. LNCS 2006 (2006) 670-675

12. Zhang, J., Tian, Y., Yang, Y., Zhu, C.: Robust foreground segmentation using subspace based background model. APCIP 20092 (Jul. 2009) 214-217

13. Li, R., Chen, Y., Zhang, X.: Fast robust eigen-background updating for foreground detection. ICIP 2006 (2006) 1833-1836

14. Yamazaki, M., Xu, G., Chen, Y.: Detection of moving objects by independent component analysis. ACCV 2006 (2006) 467-478

15. Tsai, D., Lai, C.: Independent component analysis-based background subtraction for indoor surveillance. IEEE Transactions on Image Processing, IP 20098 (January 2009) 158-167

16. Chu, Y., Wu, X., Sun, W., Liu, T.: A basis-background subtraction method using non-negative matrix factorization. International Conference on Digital Image Processing, ICDIP 2010 (2010)

17. Bucak, S., Gunsel, B.: Incremental subspace learning and generating sparse representations via non-negative matrix factorization. Pattern Recognition 42 (2009) 788-797

18. Li, X., Hu, W., Zhang, Z., Zhang, X.: Robust foreground segmentation based on two effective background models. MIR 2008 (Oct. 2008) 223-228

19. Stauffer, C., Grimson, W.: Adaptive background mixture models for real-time tracking. CVPR 1999 (1999) 246-252

20. Li, H., Jiang, T., Zhang, K.: Efficient and robust feature extraction by maximum margin criterion. Advances in Neural Information Processing Systems 16 (2004)

21. Wang, F., Zhang, C.: Feature extraction by maximizing the average neighborhood margin. CVPR 2007 (2007) 1-8

22. Yan, J., Zhang, B., Yan, S., Yang, Q., Li, H., Chen, Z.: Immc: incremental maximum margin criterion. KDD 2004 (Aug. 2004) 725-730

23. Toyama, K., Krumm, J., Brumitt, B., Meyers, B.: Wallflower: Principles and practice of background maintenance. ICCV 1999 (Sept. 1999) 255-261

24. Wren, C., Azarbayejani, A., Darrell, T., Pentland, A.: Pfinder : Real-time tracking of the human body. IEEE Transactions on PAMI 19 (July 1997) 780-785

25. Stauffer, C., Grimson, W.: Adaptive background mixture models for real-time tracking. CVPR 1999 (1999) 246-252

26. Elgammal, A., Harwood, D., Davis, L.: Non-parametric model for background subtraction. ECCV 2000 (June 2000) 751-767

27. Toyama, K., Krumm, J., Brumitt, B., Meyers, B.: Wallflower: Principles and practice of background maintenance. International Conference on Computer Vision (September 1999) 255-261

28. Chen, D., Zhang, L.: An incremental linear discriminant analysis using fixed point method. ISSN 20063971 (2006) 1334-1339

29. Kim, T., Wong, S., Stenger, B., Kittler, J., Cipolla, R.: Incremental linear discriminant analysis using sufficient spanning set approximations. CVPR (June 2007) $1-8$

30. Rosipal, R., Kramer, N.: Overview and recent advances in partial least squares. SLSFS 3940 (2005) 34-35 